\title{
On the Existence of Invariant, Absolutely Continuous Measures
}

\author{
Emil Straube
}

Mathematisches Seminar, ETH-Zentrum, CH-8092 Zürich, Switzerland

\begin{abstract}
Let $(\Omega, \mathfrak{B}, \lambda)$ be a measure space with normalized measure, $f: \Omega \rightarrow \Omega$ a nonsingular transformation. We prove: there exists an $f$-invariant normalized measure which is absolutely continuous with respect to $\lambda$ if and only if there exist $\delta>0$, and $\alpha, 0<\alpha<1$, such that $\lambda(E)<\delta$ implies $\lambda\left(f^{-k}(E)\right)<\alpha$ for all $k \geqq 0$.
\end{abstract}

In this note we consider nonsingular maps of a finite measure space into itself and give a necessary and sufficient condition for the existence of an invariant measure which is absolutely continuous with respect to the given measure. The condition says, intuitively, that the iterated inverse images of "small" sets must not become too "large". The precise formulation is given in the theorem below. The problem of existence of invariant, absolutely continuous measures arises, for example, in the study of dynamical properties of interval maps; see [1] and [2], where one finds also further references. In a different direction, we note that the investigation of invariant measures, related to the given measure in terms of their null sets, started after the discovery of the individual ergodic theorem, since only zero values of the invariant measure enter into the conclusion of the theorem, compare [3].

A measure space $(\Omega, \mathfrak{B}, \lambda)$ is a triple such that $\mathfrak{B}$ is a $\sigma$-algebra of subsets of the set $\Omega$, and $\lambda$ is a measure (positive) defined on $\mathfrak{B}$. A measurable transformation $f$ from $\Omega$ into itself is called nonsingular (with respect to $\lambda$ ) if

$$
\lambda(A)=0 \Rightarrow \lambda\left(f^{-1}(A)\right)=0, \quad \forall A \in \mathfrak{B} .
$$

A set function $v$ on $\mathfrak{B}$ is called $f$-invariant if

$$
v(A)=v\left(f^{-1}(A)\right), \quad \forall A \in \mathfrak{B} .
$$

We then have the following:

Theorem. Let $(\Omega, \mathfrak{B}, \lambda)$ be a measure space with normalized measure $\lambda, f$ a nonsingular transformation of $\Omega$ into itself. Then 
(i) there exists an f-invariant normalized measure which is absolutely continuous with respect to $\lambda$

if and only if

(ii) there exist $\delta>0$, and $\alpha, 0<\alpha<1$, such that

$$
\lambda(E)<\delta \Rightarrow \sup _{k \in \mathbb{N}} \lambda\left(f^{-k}(E)\right)<\alpha, \quad \forall E \in \mathfrak{B} .
$$

Proof of (i) $\Rightarrow$ (ii). Let $v$ be $f$ invariant, normalized and absolutely continuous with respect to $\lambda$. Choose $\delta>0$ such that $\lambda(E)<\delta$ implies $v(E)<1 / 4$. We claim that $\delta$ and $\alpha:=1-\delta / 2$ are as desired. Suppose the contrary. Then there exists $E \in \mathfrak{B}$ and an index $k$ such that $\lambda(E)<\delta$ and $\lambda\left(f^{-k}(E)\right)>1-\delta$. Thus $\lambda\left(\Omega \backslash f^{-k}(E)\right)<\delta$ and, by our choice of $\delta$,

$$
\begin{array}{r}
v(E)<1 / 4 \text { and } \\
v\left(\Omega \backslash f^{-k}(E)\right)<1 / 4 .
\end{array}
$$

But $\Omega \backslash f^{-k}(E)$ is just $f^{-k}(\Omega \backslash E)$; so the invariance of $v$ yields

$$
v(\Omega \backslash E)=v\left(f^{-k}(\Omega \backslash E)\right)=v\left(\Omega \backslash f^{-k}(E)\right)<1 / 4 .
$$

Now we have $v(E)<1 / 4$ and $v(\Omega \backslash E)<1 / 4$, which contradicts $v(\Omega)=1$.

Proof of (ii) $\Rightarrow($ i). Define the measures

We have for all $n$ :

$$
\lambda_{n}(E):=\frac{1}{n} \sum_{k=0}^{n-1} \lambda\left(f^{-k}(E)\right), \quad E \in \mathfrak{B} .
$$

and

$$
\begin{aligned}
\lambda_{n}(\Omega) & =1, \\
\lambda_{n} & \ll \lambda \quad[\text { from }(1)],
\end{aligned}
$$

$$
\lambda_{n} \geqq 0 \text {. }
$$

Taking averages in (3) has the effect that if for fixed $E \lim _{s \rightarrow \infty} \lambda_{n_{s}}(E)$ exists for a subsequence, it also exists for $\lambda_{n_{s}}\left(f^{-1}(E)\right)$ and the two limits are equal. The intuitive idea is now to try to define the measure we look for as a "suitable limit" of the $\lambda_{n}$. (Hence the averages!) To make this notion more precise, we imbed the $\lambda_{n}$ in a dual space. By (4)-(6), the $\lambda_{n}$ can be thought of as elements of the unit ball of $\mathscr{L}_{\infty}(\lambda)^{*}$, via $h \mapsto \int_{\Omega} h \cdot d \lambda_{n}, h \in \mathscr{L}_{\infty}(\lambda)$. This unit ball is weak-* compact by Alaoglu's theorem [4, V.4.2]. Let $\tilde{z}$ be a cluster point in the weak-* topology of $\mathscr{L}_{\infty}^{*}$ of the sequence $\left(\lambda_{n}\right)_{n=1}^{\infty}$. We define a set function $z$ on $\mathfrak{B}$ by

$$
z(E):=\tilde{z}\left(\chi_{E}\right), \quad E \in \mathfrak{B} .
$$

Here, $\chi_{E}$ denotes the characteristic function of the set $E$. Clearly, $z$ is finitely additive, bounded (i.e. $0 \leqq z(E) \leqq z(\Omega)=1$ ) and it vanishes on sets of $\lambda$-measure 
zero; compare [4]: IV 8.15-16, [5]: Sect. 2. Since evaluation at $\chi_{E}$ and $\chi_{f^{-1}(E)}$ are both continuous on $\mathscr{L}_{\infty}^{*}$ in the weak-* topology, there is a subsequence $\left(\lambda_{n_{s}}\right)_{s=1}^{\infty}$ such that

and

$$
z(E)=\lim _{s \rightarrow \infty} \lambda_{n_{s}}(E)
$$

$$
z\left(f^{-1}(E)\right)=\lim _{s \rightarrow \infty} \lambda_{n_{s}}\left(f^{-1}(E)\right)
$$

[Simply consider the intersections of inverse images of $1 / \mathrm{s}$-neighbourhoods of $z(E)$ and $z\left(f^{-1}(E)\right)$ respectively.] From (7), (8) and the remark following (6) we conclude that $z$ is $f$-invariant. So $z$ is a finitely additive set function vanishing on sets of $\lambda$-measure zero, invariant under $f$. Starting from $z$ we will now construct a countably additive function with these properties.

In accordance with [5, Definition 1.13], we call a finitely additive positive set function $u$ (on $\mathfrak{B}$ ) purely finitely additive if every measure $\mu$, such that $0 \leqq \mu \leqq u$, is necessarily zero. (We wish to warn the reader that in contrast to [5] we use the term "measure" only for countably additive set functions.) Then $z$ can be decomposed

$$
z=z_{c}+z_{p}
$$

with $z_{c}$ countably additive, $z_{p}$ purely finitely additive, and both positive. Moreover, this decomposition is unique. That is the content of Theorems 1.23 and 1.24 in [5]. Now we claim that $z$ is not purely finitely additive and that therefore $z_{c} \neq 0$. Otherwise, there exists a decreasing sequence of sets $E_{n} \in \mathfrak{B}$ such that

and

$$
\lim _{n \rightarrow \infty} \lambda\left(E_{n}\right)=0
$$

$$
z\left(E_{n}\right)=z(\Omega)=1
$$

[5, Theorem 1.2]. By (10), there is $n_{0}$ such that for all $n \geqq n_{0} \lambda\left(E_{n}\right)<\delta$ and, as a consequence of (ii),

$$
\sup _{k \in \mathbb{N}} \lambda\left(f^{-k}\left(E_{n}\right)\right)<\alpha
$$

where $\delta$ and $\alpha$ are chosen according to (ii).

But (12) implies that $\lambda_{k}\left(E_{n}\right)<\alpha, k=1,2,3, \ldots$; this in turn implies that $z\left(E_{n}\right) \leqq \alpha$. So we have for all $n \geqq n_{0} z\left(E_{n}\right) \leqq \alpha<1$, which contradicts (11). This proves that

$$
z_{c} \neq 0 \text {. }
$$

Next we use a fact which is not explicitly stated in [5], but which is implicit: if $u$ is a positive, finitely additive set function on $\mathfrak{B}$, then $u_{c}$ is the greatest measure among all measures $\mu$ with $0 \leqq \mu \leqq u$. This can be seen as follows: $\mu \leqq u$ implies $(u-\mu) \geqq 0$; the same is true of $(u-\mu)_{c}$ and $(u-\mu)_{p}$ re- 
spectively, by [5]: Theorems $1.23,1.24$. Writing

$$
u=\mu+(u-\mu)=\mu+(u-\mu)_{c}+(u-\mu)_{p},
$$

we find, since $\mu+(u-\mu)_{c}$ is countably additive, that $u_{p}=(u-\mu)_{p}$ and $u_{c}=\mu+(u$ $-\mu)_{c}$ [Uniqueness of the decomposition (9).] But $(u-\mu)_{c}$ is positive, therefore $\mu \leqq u_{c}$.

Applying this property of the countably additive part to the situation

$$
\begin{array}{cc}
z(E)=\underset{\uparrow}{=} z\left(f^{-1}(E)\right)=z_{c}\left(f^{-1}(E)\right)+\underbrace{z_{p}\left(f^{-1}(E)\right)}_{\geqq 0}, & \forall E \in \mathfrak{B} \\
\text { invariance of } z & (9)
\end{array}
$$

and taking into consideration that $E \mapsto z_{c}\left(f^{-1}(E)\right)$ is a countably additive set function (i.e. a measure), we get

$$
z_{c}\left(f^{-1}(E)\right) \leqq z_{c}(E), \quad \forall E \in \mathfrak{B} .
$$

Hence $E \mapsto z_{c}(E)-z_{c}\left(f^{-1}(E)\right)$ is a positive measure. Since $f^{-1}(\Omega)=\Omega$, it has total weight zero. Therefore it is the zero measure! We have shown: the countably additive part $z_{c}$ of $z$ is also invariant.

Because $z$ vanishes on sets of $\lambda$-measure zero and in view of $0 \leqq z_{c} \leqq z$ we have $z_{c} \ll \lambda$. By $(13), z_{c}$ can be normalized

$$
v(E):=z_{c}(E) / z_{c}(\Omega)
$$

to yield a measure with all the required properties.

This concludes the proof of the theorem.

Acknowledgements. I thank M. Misiurewicz and Prof. K. Osterwalder for helpful discussions.

\section{References}

1. Ruelle, D.: Commun. Math. Phys. 55, 47-51 (1977)

2. Collet, P., Eckmann, J.-P.: Theor. Phys. LN Ph 116, 331-339 (1980)

3. Rechard, O.: Duke Math. J. 23, 477-488 (1956)

4. Dunford, N., Schwartz, J.: Linear operators, Part I. New York: Interscience 1958

5. Yosida, K., Hewitt, E.: Trans. Am. Math. Soc. 72, 46-66 (1952)

Communicated by D. Ruelle

Received January 26, 1981 